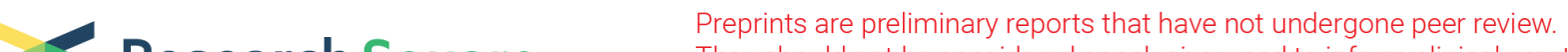 Research Square
They should not be considered conclusive, used to inform clinical practice,
or referced by the media as validated information.
}

\section{CRISPR/Cas9-based Knockout of miR-487b-3p Accelerates The Cell Proliferation of Primary Goat Myoblast by Relieving Its Inhibition of IRS1}

\section{Ming Lyu}

Northwest A\&F University: Northwest Agriculture and Forestry University

\section{Xu Wang}

Northwest A\&F University: Northwest Agriculture and Forestry University

Hongrun Qian

Northwest A\&F University: Northwest Agriculture and Forestry University

Jinrong Ma

Northwest A\&F University: Northwest Agriculture and Forestry University

\section{Yongsen Sun}

Chinese Academy of Agricultural Sciences

\section{Xiangyu Meng}

Northwest A\&F University: Northwest Agriculture and Forestry University

\section{Wenqiang Zhang}

Northwest A\&F University: Northwest Agriculture and Forestry University

\section{Zhiying Zhang}

Northwest A\&F University: Northwest Agriculture and Forestry University

Kun Xu ( $\nabla$ xukunas@nwafu.edu.cn)

Northwest A\&F University: Northwest Agriculture and Forestry University https://orcid.org/0000-00015714-9090

\section{Research}

Keywords: miR-487b-3p, CRISPR/Cas9, Primary goat myoblast, Proliferation

Posted Date: March 12th, 2021

DOl: https://doi.org/10.21203/rs.3.rs-277165/v1

License: (c) (1) This work is licensed under a Creative Commons Attribution 4.0 International License. Read Full License 


\section{Abstract}

Background: MicroRNAs (miRNAs) are endogenously expressed small noncoding RNAs with a length of about 22 nucleotides and play critical roles in the regulation of posttranscriptional gene expression. miR$487 \mathrm{~b}-3 \mathrm{p}$ has been recently identified as a key regulator in skeletal muscle growth and development. However, the function of miR-487b-3p on goat skeletal muscle remains to be investigated.

Results: In this study, we found that miR-487b-3p was widespread in goat different tissues, with a significantly higher expression in muscle, especially in lamb. The results demonstrated that the expression of miR-487b-3p was gradually up-regulated during myoblast proliferation. Then, miR-487b-3p knockout primary goat myoblast cells clones were obtained by using CRISPR/Cas 9 system and our RPG surrogate reporter-based screening. Further investigation uncovered that the knockout of miR-487b-3p significantly accelerated primary goat myoblast proliferation, which was accompanied by up-regulation of cell cycle-related genes, such as PCNA, Cyclin E and CDK2. What's more, we found that miR-487b-3p targets directly the 3'UTR of insulin receptor substrate 1 (IRS1) gene and IRS1 knockdown by siRNA was able to down-regulate the expression of the cell cycle-related genes.

Conclusions: Collectively, these founding demonstrated miR-487b-3p as a potent inhibitor of cell proliferation which functions by targeting IRS1 gene in primary goat myoblast.

\section{Background}

Skeletal muscle is the most abundant tissue in mammals and plays an important role in body metabolism [1]. During the formation of skeletal muscle, mononucleated myoblast expand, migrate, and differentiate into myoblast, which is a complex process regulated by a group of myogenic regulator factors (MRF). These regulators, including Pax3, Pax7, Myf5 and MyoG, each of which plays an essential role in skeletal muscle development [2;3]. Therefore, further understanding of the skeletal muscle development process and related molecular mechanisms in goat will contribute to improve the growth of young lambs and facilitate the breeding mutton goats.

MicroRNAs (miRNAs) are a class of evolutionarily conserved small RNAs that inhibit target gene expression at a post-transcriptional regulation either by degrading or arresting the translation of specific messenger RNAs (mRNAs). The short single-stranded miRNA usually binds to the 3 ' untranslated region (3'UTR) of specific mRNA based on sequence homology [4;5], resulting in the degradation of target mRNA or the repression of corresponding protein translation [6]. Up to now, a series of miRNAs have been verified to be involved in the regulation of myoblast proliferation and differentiation. Among which, miR-1 [7], miR-133 [8], miR-206 and miR-486 [9] are key muscle specific regulators involved in muscle myogenesis. In addition, skeletal muscle is also enriched with many ubiquitously expressed miRNAs, including miR-24 [10], miR-29 [11], miR-125b [12] miR-181 [13], and miR-214 [14], which are also necessary for the regulation of muscle development. Both muscle-specific and ubiquitously expressed miRNAs have been demonstrated as major regulators of fundamental biological processes and play 
essential roles in myogenesis [15]. However, further study of functional miRNAs in goat muscle development, although which is just a small fraction of the many thousands of miRNAs, remains necessary to improve our understanding how miRNA net-work regulates goat myogenesis.

Previous studies have reported that the cluster $14 q 32.31$ member miR-487b-3p is ubiquitously expressed and plays important roles in many biological processes, such as cell proliferation, differentiation, and disease [16; 17]. miR-487b binds directly to both rat and human insulin receptor substrate 1 (IRS1) 3'UTR and inhibits reporter gene expression in primary rat and human arterial adventitial fibroblasts $[16 ; 18]$. IRS1 is one of the dominant acting regulators of cell proliferation and survival. Recent study showed that miR-487b-3p expression varies from different stages of goat muscle development and myogenesis, and could delay proliferation and differentiation in mouse myoblast-derived $\mathrm{C} 2 \mathrm{C} 12$ cells [16]. However, to our knowledge, the function of miR-487b-3p on goat myogenesis remains unknown.

Synthetic small interfering RNAs (siRNAs) are commonly used to knock-down target RNAs to explore their functions, including mRNAs, IncRNAs, miRNAs [19;20] and so on. Despite it's fast and cost efficient, RNA interference (RNAi) technique suffers from limited short-term inhibition and inconsistent silencing efficiency. The CRISPR (clustered regularly interspaced short palindromic repeats)/Cas9 (CRISPRassociated protein 9) technology has been widely used for genome editing in various cell types and organisms since its advent [21]. With robust on-target activity and high fidelity, CRISPR/Cas9 technology can be used to knock-out interested miRNA by introducing indels into its seed sequence, and has become a more preferred tool than RNAi in the study of miRNA gene function [22]. Here, to investigate the role of miR-487b-3p in goat myogenesis, we created miR-487b-3p knockout (KO) primary goat myoblast cell clones by using CRISPR/Cas9 system and our DsRed-Puror-eGFP (RPG) surrogate reporter-based screening [23-25]. Further experimental investigation demonstrated miR-487b-3p as a potent inhibitor of cells proliferation and that the knockout of miR-487b-3p significantly accelerated primary goat myoblast proliferation. In summary, this study provides new insight into the function of miR-487b-3p in primary goat myoblast, which will expand our understanding of its role in the regulation of skeletal muscle growth.

\section{Methods}

\section{Goat tissue samples and primary myoblast cells}

The goats were raised in strict accordance with the guides of Northwest Agricultural and Forestry University (NWAFU) Animal Care and Use Committee. Heart, liver, spleen, lung, kidney, and muscle tissue samples were collected from three lamb goat ( 90 days old) and three adult goats ( 2 years old). Primary myoblast cells were isolated from skeletal muscle tissues of the lamb goat, and were purified and cultured according to previously described protocol [26]. Briefly, cells were cultured in growth medium containing DMEM/F12 basic medium (C11330500BT, Invitrogen Corp, Waltham, MA, USA), 20\% fetal bovine serum (FBS) (10099-141C, Invitrogen Corp., Waltham, MA, USA), and antibiotics (100 U/mL 
penicillin and $100 \mathrm{~g} / \mathrm{mL}$ streptomycin) (15140-122, Invitrogen Corp., Waltham, MA, USA) in $5 \% \mathrm{CO}_{2}$ at $37^{\circ} \mathrm{C}$, with the medium changed every day.

\section{RNA Isolation and real-time quantitative PCR (RT-qPCR)}

Total RNA was extracted from goat tissue samples or cultured cells using the reagent Trizol (9109, Takara). The concentration of total RNA measured by NanoDrop2000 (Thermo scientific) and the quality was checked by denaturing agarose gel electrophoresis. The cDNA synthesis was performed with reverse transcription kits (RR047A, Perfect Real Time, Takara). For quantification of miR-487b-3p expression, miRNA specific complementary DNA was generated using miRNA stem-loop-specific primers (Table 1). The cDNA generated was stored at $-20^{\circ} \mathrm{C}$ for subsequent usage.

RT-qPCR was performed using TB green premix Ex taq II (RR820A, Perfect Real Time, Takara) on a Light Cycler 96 real-time system (Roche) with a $25 \mu \mathrm{L}$ reaction volume. Each sample was carried out in triplicate and was repeated for three times at least. The mRNA expression levels of all coding genes were normalized to the housekeeping gene glyceraldehyde-3-phosphate dehydrogenase (GAPDH), which was used as an internal standard. On the other hand, the expression level of miR-487b was quantified with $18 \mathrm{~S}$ RNA as the reference. The relative gene expression was analyzed using the comparative $\mathrm{Ct}\left(2^{-} \triangle \triangle \mathrm{Ct}\right)$ method.

\section{Construction of CRISPR/Cas9 and RPG surrogate reporter vectors}

In order to knockout miR-487b, three top single guide RNAs (sgRNAs) were designed according to the genomic sequence of Capra hircus miR-487b-3p (chi-miR-487b-3p) using the CRISPR Design Tool (http://chopchop.cbu.uib.no/) based on the following criteria: the sgRNA should have a high efficiency score and low off-target effects. Single-strand DNA annealing oligonucleotides for the guide and the target sequences of the three sgRNAs were synthesized by Invitrogen (Shanghai, China, as shown in table 2). Then, Annealed oligonucleotides for the three sgRNA guides were cloned into the $B b s$ I sites of the plasmid pX330-U6-Chimeric_BB-CBh-hSpCas9 (pX330) (plasmid \#42230, Addgene, Cambridge, MA) to construct the corresponding sgRNA/Cas9 expression vectors. The SSA-RPG surrogate reporter vectors was designed and constructed as we previously reported [24]. Generally, the oligo-nucleotide annealed products with PAMs for the three sgRNA targets were inserted into the Not I/ BamHI sites of the SSA-RPG parental vector to generate the corresponding surrogate reporter vectors, respectively. All the plasmid vectors were confirmed by sequencing.

\section{Cell transfection, screening and T7E1 cleavage assays}

Primary goat myoblast cells $\left(1 \times 10^{6}\right.$ cells/well) were seeded into 6-well plates and cultured in normal culture medium to reach $80 \%$ of confluence. The myoblast cells were transiently transfected with $3 \mu \mathrm{g}$ plasmid DNA ( $2 \mu \mathrm{g}$ sgRNA/Cas 9 expression vector and $1 \mu \mathrm{g}$ RPG surrogate reporter vector) using Lipofectamine 3000 reagent (L3000008, Invitrogen, Waltham, MA) following to the manufacturer's protocol. The parental vector pX330 without sgRNA guide sequence served as a negative control. The 
medium was changed 4 hours after transfection and the cells were screened for another 48 hours with $1.0 \mu \mathrm{g} / \mathrm{mL}$ of puromycin (P8833, Sigma) supplemented. Surviving cells were expanded and the genomic DNAs were extracted using the E.Z.N.A.DNA Kit (OMEGA Bio-Tek) for PCR and T7E1 cleavage assay. PCR was conducted using the $2 \times$ Taq Master Mix (Novoprotein). The DNA fragments spanning target sites were amplified with the primers: $F$ 5'-GACCCAGTCCACATAC AGCAAG-3', R 5'-

CGATAATCGCATCACCATTAC-3'. Purified PCR products were subject to T7E1-cleavage assay (M0302L, NEB, Ipswich, MA, USA), and the digested DNA fragments were detected by gel electrophoresis. The band intensity for different DNA fragments was measured by Image $\mathrm{J}$ software (Image Lab, http://imagej.net) and was used for calculating the indel frequencies within the target sites as reported previously [27]. The purified PCR products were also inserted into the PMD ${ }^{\mathrm{TM}}$ 19-T Vector (Takara) by TA cloning for sequencing verification.

In addition, for chemically synthesized siRNAs, cells were transfected at 50\% density with $50 \mathrm{nM}$ RNA using lipofectamine 3000. The siRNA sequences are shown in Supplementary table S1.

\section{Myoblast cells clone detection and off-target effect analysis}

After puromycin resistant screening, selected myoblast cells were seeded into a 96-well plate for single cell cloning. After 10-15 days further culture, the cells were collected with half of each cell clones were seeded into new 48-well plates and the remaining half for genomic DNA extraction. Then, the precursor sequence of miR-487b-3p were amplified by PCR for validating the genomic modification by sequencing.

To assess the off-target effects, the potential off-target sites (POTs) for each sgRNA were predicted according to an online design tool (http://crispr.mit.edu/) and Cas-OFFinder

(http://www.rgenome.net/cas-offinder/). The genomic sequences of the POTs were also amplified by PCR for sequencing verification. The primer sequences are shown in Supplementary table S2.

\section{Cell counting kit-8 (CCK-8) and EdU imaging assays}

The miR-487b-3p KO myoblast cells were seeded at a density of $1 \times 10^{3}$ cells per well in a 96 well plate in growth medium. Six independent biological replicates for each treatment were conducted. After the maintained for 24 hours, the cells were switched to the medium with $10 \%$ Cell Counting Kit-8 (CCK- 8 ) reagent (EQ829, DOJINDO) and were incubated for another 4 hours at $37^{\circ} \mathrm{C}$, which was followed by the absorbance measurement at $450 \mathrm{~nm}$ using a SYNERGY/H1 microplate reader (BioTek). The intensity of the color was directly proportional to the number of viable cells in the sample.

In addition, EdU imaging assay was carried out using the 488 EdU Click Proliferation kit (Beyotime) according to the manufacturer's instruction. Myoblast cells were incubated with EdU (50 mM) (Beyotime) in the culture medium for 2 hours. Following incorporation of EdU and fixation, myoblast cells were subjected to Click-iT reaction (Invitrogen) for 30 minutes in the dark to add biotin to the EdU, and the cell nuclei were stained with Hoechst 33342 for 10 minutes. Afterward, the cells were visualized by using a 
Cell imaging multifunctional detection system (BioTek) and the data were analyzed with Image $\mathrm{J}$ software.

\section{Flow cytometric analysis}

The miR-487b-3p KO myoblast cells were seeded in 6-well plates at a density of $5 \times 10^{5}$ cells per well. After 48-hour culture, cells were washed three times with PBS and harvested. The cells were collected and fixed in cold $70 \%$ ethanol at $4{ }^{\circ} \mathrm{C}$ overnight. After RNase A treatment $(1 \mathrm{mg} / \mathrm{mL})$ at $37^{\circ} \mathrm{C}$ for 30 minutes, and then staining with 4,6-diamidino-2-phenylindole (DAPI) staining solution (Solarbio) $(50 \mu \mathrm{g} / \mathrm{mL} \mathrm{DAPI}, 0.2 \%$ Triton X-100, $100 \mu \mathrm{g} / \mathrm{mL}$ RNase, PBS) for 1 hour at $4^{\circ} \mathrm{C}$ in the dark. Then the cell suspension was centrifuged at $1200 \mathrm{rpm}$ for 5 minutes, and the supernatant was discarded. Finally, the cells were resuspended in $1 \mathrm{~mL}$ PBS and analyzed by a BD FACSAria ${ }^{\mathrm{TM}}$ III flow cytometry system (BD).

\section{Dual-luciferase reporter assay}

The 3'UTR of goat IRS1 mRNA were amplified from myoblast cells CDNA with the primers as shown in table 2. The wild type or mutant 3'UTR sequences of IRS1 were cloned into the psi-CHECK2 vector (Promega, Germany) with restriction sites of Xho I and Not I, respectively. HEK293T cells were seeded in 48-well culture plates at a density of $1 \times 10^{4}$ cells per well. The cells were co-transfected with $250 \mathrm{ng}$ of the wild-type (WT, psi-CHECK2-IRS1-3'UTR) or the mutant (Mutant, psi-CHECK2-IRS1-3'UTR-mut) 3'UTR plasmids and $100 \mathrm{nM}$ miR-487b-3p mimics or negative control (NC) using the Lipofectamine 3000 reagent (L3000015, Invitrogen, Waltham, MA) following the manufacturers protocol. After the transfection for 48 hours, cells were harvested and lysed in passive lysis buffer (Promega, USA), and the dualluciferase activity assay was performed according to the manufacturer's instruction (Promega, USA).

\section{miRNA target gene prediction and analysis}

The sequences of miRNAs were obtained from the miRNA Registry miRBase (http://www.mirbase.org/) and the 3'UTR sequence of IRS1 were downloaded from NCBI (https://www.ncbi.nlm.nih.gov/). The target genes of miRNA were predicted by TargetScan (http://www.targetscan.org), miRDB (http://www.mirdb.org/miRDB/) and David Bioinformatics analysis (https:// david.ncifcrf.gov/).

\section{Protein extraction and western blot analysis}

The total protein was extracted using RIPA lysis buffer (R0010, Solarbio, Beijing, China) with a protease inhibitor mix (04693132001, Roche Diagnostics, Ltd., Mannheim, Germany) after washing the cells with PBS three times. The protein concentration was determined using BCA Protein Assay kit (23227, Thermo Fisher Scientific, Rockford, IL). $30 \mu \mathrm{g}$ of protein for each sample was separated by SDS-PAGE (sodium dodecyl sulfate polyacrylamide gel electrophoresis) followed by transferring onto PVDF (polyvinylidene fluoride) membrane (HATF00010, Millipore, Burlington, MA). The membrane was blocked with $5 \%$ defatted milk (232100, BD, Franklin Lakes, NJ) in TBST (Tris-buffered saline with Tween 20) buffer for 1 hours at room temperature, and then was incubated with antibodies (1:1000) against Proliferating cell 
nuclear antige (PCNA) (Abcam), Cyclin dependent kinases 2 (CDK2) (SAB), Cyclin E, p27 (Santa Cruz) and Glyceraldehyde-3-phosphate dehydrogenase (GAPDH) (diluted 1:2000) (Bioss) at $4^{\circ} \mathrm{C}$ overnight. The following day each membrane was washed three times with TBST, for 10 minutes each time.

Subsequently, the membrane was incubated with HRP-conjugated secondary antibodies (anti-mouse IgG or anti-rabbits $\lg G$ ) (Bioss) diluted at 1:2000. Chromogenic reaction was performed using an enhanced chemiluminescent (ECL) western blotting substrate substrate (K-12045-D10, Advansta, California, USA) and was detected by Sage Capture TM System (BioTek). Image J software was used for densitometric analysis.

\section{Statistical analysis}

All statistics were analyzed by GraphPad Prism 6.0 software and the data was expressed as the "mean \pm SEM" of at least three independent repeats. The statistical significance of difference was assessed by unpaired Student's t-test for two group comparisons and a one-way ANOVA for more than two groups. The difference was considered significant when the corresponding $P$ value was less than $0.05\left(^{*}\right)$ or 0.01 $(\star *)$.

\section{Results}

\section{miR-487b-3p acts as a candidate regulator in goat skeletal myogenesis}

To address the function of goat miR-487b-3p, the expression pattern of miR-487b in different tissues was detected by RT-qPCR analysis. The data showed that miR-487b-3p was enriched in skeletal muscle compared with heart, liver, spleen, lung and kidney during lamb-stage and adult-stage (Fig. 1a, and b). Besides, we found a higher expression level in lamb goat than adult goat (Fig. 1c). Significant upregulation of miR-487b-3p during myoblast proliferation was further observed (Fig. 1d). Bioinformatic analysis found that miR-487b-3p is located on Chromosome 21 and is composed of 22 nucleotides. The mature miR-487b-3p sequence is highly conserved among several species, including Ovis aries, Bos taurus, Mus musculus, Sus scrofa and Homo sapiens, according to the miRBase database (www.mirbase.org/) and UCSC (http://genome.ucsc.edu/) (Fig. 1e). These results suggested that miR$487 b-3 p$ might play a critical role in goat myogenesis.

\section{RPG-based screening for genetically targeted goat myoblast cells}

Three sgRNAs were designed to target the genome sequence of miR-487b-3p. The location of the three sgRNAs (sg1, sg2, sg3) is shown in Figure 2A with sg1 and sg2 targeting the seed sequence of miR-487b$3 p$. Then the three sgRNAs were cloned individually into the pX330 vector with Cas 9 expression cassette (Fig. 2c) and were used to transfect goat myoblast cells. The frequencies of indels induced by the three sgRNAs were assessed using an in vitro digestion assay. The result suggested a much higher cleavage activity of sg2 with $12.5 \%$ indel frequency than sg1 and sg3 (Fig. 2b). 
To enrich the genetically targeted goat myoblast cells by RPG surrogate reporter-based screening, we developed the RPG surrogate reporter system as we previously reported (Fig. 2c). After co-transfected with the CRISPR/Cas9 and RPG surrogate reporter vectors, the primary goat myoblast cells were subject to puromycin selection with the minimal lethal dose $0.75 \mu \mathrm{g} / \mathrm{mL}$ (Suppl. Figure 1). Further genotyping analysis of the screened cells by the digestion assay demonstrated $8.3 \%, 23.3 \%$ and $8.9 \%$ indel frequencies for sg1, sg2 and sg3, respectively (Fig. 2d). The genomic indels with random sizes adjacent to PAM sequence from the sg2 experiment group were further confirmed by TA cloning and subsequent sequencing (Fig. 2e). These results indicated that our RPG surrogate reporter system can be used for highly efficient enrichment of genetically targeted goat myoblast cells.

\section{miR-487b-3p KO cell clones and off-target effect analyses}

Several miR-487b-3p KO primary goat myoblast cell clones from the sg2 experiment group were obtained and expanded after the RPG surrogate reporter-based screening. The miR-487b-3p genomic loci of these cell clones were examined by PCR and sequencing. One of the cell clones with a deletion of 7 nucleotides (Fig. 3a) was chosen for off-target effect and further function analyses. T7E1 assay demonstrated that the indel frequency for expanded cell clone was $35.9 \%$ (Fig. 3b). For off-target analysis, only 1 potential off-target site for sg2 was predicted by both the online design tool (http://crispr.mit.edu/) and CasOFFinder (Fig. 3c). The genomic locus of the off-target site was amplified by PCR and subjected to sequencing and T7E1 cleavage analyses (Fig. 3d). The sequencing result demonstrated no visible mutant peaks. On the other hand, T7E1 cleavage assay also showed no difference between the wild type and miR-487b-3p KO cells (Fig. 3e). In conclusion, these results suggested no detectable at the potential offtarget site in the miR-487b-3p KO cell clone, which could be used for further function analysis.

\section{Knockout of miR-487b-3p promotes goat myoblast cells proliferation}

As for the function analysis, several assays were conducted to explore the effect of miR-487b-3p knockout on goat myoblast cells. Firstly, the barely detectable expression of miR-487b-3p in its KO cell clone was confirmed by RT-qPCR (Fig. 4a). Secondly, significantly up-regulated expression of cell cyclerelated genes, including PCNA, Cyclin E and CDK2, were detected in miR-487b-3p KO cells at both the mRNA (Fig. 4a) and protein levels (Fig. 4b). While the cell cycle inhibitor gene p27 was down-regulated in the opposite.

Next, the EdU incorporation assay demonstrated that the miR-487b-3p KO group had more labeled cells than that in the wildtype control group (Fig. 4c). The CCK-8 assay result also suggested that miR-487b-3p $\mathrm{KO}$ cells possess significantly higher viability compared with the wildtype control (Fig. 4d). What's more, the flow cytometric analysis result with more S-phage cells in the miR-487b-3p KO group (Fig. 4e) further revealed that the knockout of miR-487b-3p could promote the cell cycle process. Taken together, all these results indicated that the knockout of miR-487b-3p could promote the proliferation of goat myoblast cells. 
To explore the molecular mechanism of miR-487b-3p in goat myoblast, its potential target genes were predicted using the online miRBase and TargetScan software, and the IRS1 gene was chosen as the preferred candidate for further study (Fig. 5a). Strikingly, we found that miR-487b-3p and IRS1 had completely opposite expressing profiles during proliferating goat myoblast cells (Fig. $1 \mathrm{~d}$ and $5 \mathrm{~b}$ ), and 100 nM miR-487b-3p mimics was enough to inhibit the IRS1 mRNA expression by 50\% (Fig. $5 \mathrm{c}$ and d).

Next, the dual-luciferase reporter system was constructed to investigate whether the IRS1 gene is a direct target of miR-487b-3p. The 3'UTR fragments from IRS1 gene containing the predicted wildtype or mutant miR-487b-3p binding sites were cloned into the psi-CHECK-2 vector as shown in Figure 5A. As expected, the relative luciferase activity of the wildtype $3^{\prime} U T R$ group was significantly inhibited as responded to miR-487b-3p mimics ( $P<0.05$, Fig. 5e). In return, the IRS1 expression was significantly increased at both the mRNA and protein levels in miR-487b-3p KO cells (Fig. $5 f$ and g). Altogether, these results provide solid validation that IRS1 gene is a direct downstream target of miR-487b-3p in goat myoblast cells.

\section{Knockout of miR-487b-3p affects myoblast proliferation by targeting IRS1}

To further investigate the function of IRS1 gene in miR-487b-3p KO goat myoblast cells, the siRNA rescue experiment was conducted. miR-487b-3p KO goat myoblast cells were transfected with silRS1 against IRS1 gene or the negative control siNC. The RT-qPCR and western blot detection results showed that the IRS1 mRNA and protein expression were both decreased by the transfection of silRS1 (Fig. 6a and b).

Further detection results demonstrated that the knockdown IRS1 gene by silRS1 in the miR-487b-3p KO cells also caused altered expression of cell cycle-related genes. The expression of PNCA, Cyclin E and $C D K 2$ genes were reduced, while the $p 27$ gene increased, in the levels of both mRNA and protein (Fig. $6 \mathrm{c}$ and d). These results were just the opposite of those detected when no siRNA rescued as shown in Figure 4. We therefore could conclude that miR-487b-3p was a negative regulator to inhibit goat myoblast cells proliferation by targeting IRS1 gene.

\section{Discussion}

Recently, an increasing number of reports indicate that miRNAs play important roles in the regulation of animal myogenesis $[10 ; 19 ; 28]$. miR-487b-3p has been reported by previous study with high-throughput sequencing and RNAi to modulate myoblast cells proliferation in different species. This study was aimed to investigate the effect of miR-487b-3p on the cell proliferation of primary goat myoblast. The expression profile of miR-487b-3p in different lamb or adult goat tissues was firstly illustrated. Then, its association with the cell proliferation of goat myoblast was further verified. Bioinformatics analysis found that the seed sequence of goat miR-487b-3p is identical between Ovis aries, Bos taurus and other species, suggesting important biological role of miR-487b-3p in regulation of evolutionarily conservative genes.

As a simple but efficient site-directed genome editing technology, CRISPR/Cas9 system has been widely used for genome editing in multiple organisms. However, the low transfection efficiency of primary cells 
and the difficulty to select positive cell clones have always been the challenges in animal gene editing research [32;33]. To enrich the genetically targeted goat myoblast cells, the RPG surrogate reporter system was constructed as we previously reported. The gene targeting efficiency in primary goat myoblast cells was significantly improved, and miR-487b-3p KO cell clones were obtained successfully by our RPG surrogate reporter-based screening. One of the cell clones with a deletion of 7 nucleotides in miR$487 b-3 p$ seed sequence verified with no detectable off-target effect was chosen for further function analysis.

Previous research has reported that the knockdown of miR-487b-3p by RNAi could suppresses the proliferation and differentiation of $\mathrm{C} 2 \mathrm{C} 12$ myoblast [16]. In this study, we succeeded with the knockout of miR-487b-3p in goat myoblast cells by introducing indels into its seed sequence using CRISPR/Cas9. Both the results of EdU and CCK-8 assays demonstrated that the knockout of miR-487b-3p could accelerate the cell proliferation of primary goat myoblast cells. Consistently, the cell cycle analysis showed the G1-phase and G2-phase miR-487b-3p KO cells were decreased compared with wildtype control, while the S-phase KO cells was increased. A variety of molecules have been recognized to mediate the cell cycle progression. PCNA, Cyclin E, CDK2 and $p 27$ are important regulators of the G1-S transition, which function to modulate the activity of cyclin-dependent kinases [34; 35]. The expression of these cell cycle related-genes was significantly altered as anticipated at both the mRNA and protein levels in the miR-487b-3p KO goat myoblast cells. Altogether, these results point to an essential role of miR$487 b-3 p$ in regulating the cell proliferation of goat myoblast cells.

IRS1 is the most important representative of the IRS protein family and the critical factor in insulin/IGF1signaling pathway [36; 37]. Here, we identified IRS1 as the target gene of miR-487b-3p in goat myoblast cells by bioinformatics analysis, RT-qPCR, western blot and dual-luciferase reporter assays. The IRS1 gene expression declined after the treatment with miR-487b-3p mimics and the expression profile showed an inverse-correlation with that of miR-487b-3p during goat myoblast proliferation. It has been already acknowledged that IRS1 is a critical mediator of PI3K/Akt and AMPK signaling in the regulation of muscle growth and metabolism [38; 39]. As expected, the expression of IRS1 gene and the cell cyclerelated genes including PCNA, Cyclin E and CDK2 was significantly increased in the miR-487b-3p KO goat myoblast cells. On the contrary, silRS1 could rescue the inhibition of IRS1 and these cell cycle-related genes in miR-487b-3p KO cells. These results suggested that miR-487b-3p targets the IRS1 gene directly at its 3'UTR, and the knockout of miR-487b-3p accelerates the cell proliferation of primary goat myoblast cells by relieving its inhibition of IRS1.

\section{Conclusions}

In summary, we succeed in obtaining the cell clones of miR-487b-3p KO primary goat myoblast by using CRISPR/Cas9 system and RPG surrogate reporter-based screening. miR-487b-3p targets IRS1 gene directly and the knockout of miR-487b-3p accelerates the cell proliferation of primary goat myoblast. These results provide novel insight into the function of miR-487b-3p in goat myoblast cells and will contribute to our understanding of the growth mechanism of goat skeletal muscle. 


\section{Abbreviations}

IRS1: Insulin receptor substrate 1 gene; PCNA: Proliferating cell nuclear antige; CDK2: Cyclin dependent kinases 2; DAPI: 4',6-diamidino-2-phenylindole; MRF: Myogenic regulator factors; 3'UTR: 3' untranslated region; RNAi: RNA interference; siRNAs: Synthetic small interfering RNAs; RT-qPCR: Real-time quantitative PCR. CRISPR: clustered regularly interspaced short palindromic repeats; Cas9: CRISPR-associated protein 9; RPG: DsRed-Puror-eGFP Surrogate reporter-based screening; GAPDH: Glyceraldehyde-3-phosphate dehydrogenase; SgRNAs: Single guide RNAs; POTs: Potential off-target sites;

\section{Declarations}

\section{Acknowledgments}

We thank all the members of the Animal genome editing Laboratory of the College of Animal Science and Technology, Northwest A\&F University who make efforts to these experiments.

\section{Authors contributions}

Ming Lyu, Zhiying Zhang. and Kun Xu conceived and designed the experiments; Ming Lyu., Xu Wang and Xiangyu Meng performed the experiments; Ming Lyu., Hongrun Qian., Jinrong Ma., and Yongsen Sun analyzed and interpreted the data; Ming Lyu., Kun Xu. and Wenqiang Zhang assisted data analysis and interpretation; Ming Lyu and Kun Xu wrote the manuscript. All authors approved the final version of the manuscript.

\section{Finding}

This work was supported by grants from the China Postdoctoral Science Foundation (2018T111111 and 2015M580887), the National Natural Science Foundation of China (NSFC, 31702099).

\section{Availability of data and materials}

The data sets used and/or analyzed during the current study are available from the corresponding author on reasonable request.

\section{Ethics approval and consent to participate}

All the animal procedures were raised in strict accordance with the guides of Northwest Agricultural and Forestry University (NWAFU) Animal Care and Use Committee. The experimental protocol was approved by the Departmental Animal Ethics Committee of Northwest A\&F University (14-233, 10 December 2014).

\section{Consent for publication}

Not applicable. 


\section{Competing interests}

The authors have declared no conflict of interest.

\section{References}

1. Verma $M$, Asakura $Y$, Murakonda BSR, Pengo T, Latroche $C$, Chazaud $B$, et al. Muscle satellite cell Cross-Talk with a vascular niche maintains quiescence via VEGF and notch signaling. Cell stem cell. 2018;23(4):530-43. doi:10.1016/j.stem.2018.09.007.

2. Liu Y, Chu A, Chakroun I, Islam U, Blais A. Cooperation between myogenic regulatory factors and SIX family transcription factors is important for myoblast differentiation. Nucleic acids research. 2010;38(20):6857-71. doi:10.1093/nar/gkq585.

3. Hindi SM, Shin J, Gallot YS, Straughn AR, Simionescu-Bankston A, Hindi L, et al. MyD88 promotes myoblast fusion in a cell-autonomous manner. Nature communications. 2017;8(1):1624. doi:10.1038/s41467-017-01866-w.

4. Kooshapur H, Choudhury NR, Simon B, Mühlbauer M, Jussupow A, Fernandez N, et al. Structural basis for terminal loop recognition and stimulation of pri-miRNA-18a processing by hnRNP A1. Nature communications. 2018;9(1):2479. doi:10.1038/s41467-018-04871-9.

5. Kim H, Kim J, Kim K, Chang H, You K, Kim VN. Bias-minimized quantification of microRNA reveals widespread alternative processing and 3 ' end modification. Nucleic acids research. 2019;47(5):2630-40. doi:10.1093/nar/gky1293.

6. Siciliano V, Garzilli I, Fracassi C, Criscuolo S, Ventre S, di Bernardo D. MiRNAs confer phenotypic robustness to gene networks by suppressing biological noise. Nature communications. 2013;4:2364. doi:10.1038/ncomms3364.

7. Yang B, Lin H, Xiao J, Lu Y, Luo X, Li B, et al. The muscle-specific microRNA miR-1 regulates cardiac arrhythmogenic potential by targeting GJA1 and KCNJ2. Nature medicine. 2007;13(4):486-91.

8. Yin H, Pasut A, Soleimani VD, Bentzinger CF, Antoun G, Thorn S, et al. MicroRNA-133 controls brown adipose determination in skeletal muscle satellite cells by targeting Prdm16. Cell Metabol. 2013;17(2):210-24. doi:10.1016/j.cmet.2013.01.004.

9. Dey BK, Gagan J, Dutta A. MiR-206 and - 486 induce myoblast differentiation by downregulating Pax7. Molecular cellular biology. 2011;31(1):203-14. doi:10.1128/MCB.01009-10.

10. Sun Q, Zhang Y, Yang G, Chen X, Zhang Y, Cao G, et al. Transforming growth factor-beta-regulated miR-24 promotes skeletal muscle differentiation. Nucleic acids research. 2008;36(8):2690-9. doi:10.1093/nar/gkn032.

11. Wang H, Wang B, Zhang A, Hassounah F, Seow Y, Wood M, et al. Exosome-Mediated miR-29 transfer reduces muscle atrophy and kidney fibrosis in mice. Molecular therapy: the journal of the American Society of Gene Therapy. 2019;27(3):571-83. doi:10.1016/j.ymthe.2019.01.008.

12. Ge Y, Sun Y, Chen J. IGF-II is regulated by microRNA-125b in skeletal myogenesis. J Cell Biol. 2011;192(1):69-81. doi:10.1083/jcb.201007165. 
13. Naguibneva I, Ameyar-Zazoua M, Polesskaya A, Ait-Si-Ali S, Groisman R, Souidi M, et al. The microRNA miR-181 targets the homeobox protein Hox-A11 during mammalian myoblast differentiation. Nat Cell Biol. 2006;8(3):278-84.

14. Juan AH, Kumar RM, Marx JG, Young RA, Sartorelli V. Mir-214-dependent regulation of the polycomb protein Ezh2 in skeletal muscle and embryonic stem cells. Molecular cell. 2009;36(1):61-74. doi:10.1016/j.molcel.2009.08.008.

15. Mori MA, Ludwig RG, Garcia-Martin R, Brandão BB, Kahn CR. Extracellular miRNAs: From biomarkers to mediators of physiology and disease. Cell Metabol. 2019;30(4):656-73. doi:10.1016/j.cmet.2019.07.011.

16. Wang J, Tan J, Qi Q, Yang L, Wang Y, Zhang C, et al. MiR-487b-3p suppresses the proliferation and differentiation of myoblasts by targeting IRS1 in skeletal muscle myogenesis. Int J Biol Sci. 2018;14(7):760-74. doi:10.7150/ijbs.25052.

17. Yi H, Geng L, Black A, Talmon G, Berim L, Wang J. The miR-487b-3p/GRM3/TGF $\beta$ signaling axis is an important regulator of colon cancer tumorigenesis. Oncogene. 2017;36(24):3477-89. doi:10.1038/onc.2016.499.

18. Nossent AY, Eskildsen TV, Andersen LB, Bie P, Brønnum H, Schneider M, et al. The 14q32 microRNA487b targets the antiapoptotic insulin receptor substrate 1 in hypertension-induced remodeling of the aorta. Annals of surgery. 2013;258(5):743-51. doi:10.1097/SLA.0b013e3182a6aac0. 752-3.

19. Ma M, Wang X, Chen X, Cai R, Chen F, Dong W, et al. MicroRNA-432 targeting E2F3 and P55PIK inhibits myogenesis through PI3K/AKT/mTOR signaling pathway. RNA Biol. 2017;14(3):347-60. doi:10.1080/15476286.2017.1279786.

20. Li Z, Cai B, Abdalla BA, Zhu X, Zheng M, Han P, et al. LnclRS1 controls muscle atrophy via sponging miR-15 family to activate IGF1-PI3K/AKT pathway. Journal of cachexia sarcopenia muscle. 2019;10(2):391-410. doi:10.1002/jcsm.12374.

21. Cong L, Ran FA, Cox D, Lin S, Barretto R, Habib N, et al. Multiplex genome engineering using CRISPR/Cas systems. Science (New York, N.Y.). 2013;339(6121): 819-23. doi:10.1126/science.1231143.

22. Pickar-Oliver A, Gersbach CA. The next generation of CRISPR-Cas technologies and applications. Nat Rev Mol Cell Biol. 2019;20(8):490-507. doi:10.1038/s41580-019-0131-5.

23. Xu K, Ren C, Liu Z, Zhang T, Zhang T, Li D, et al. Efficient genome engineering in eukaryotes using Cas9 from Streptococcus thermophilus. Cell Mol Life Sci. 2015;72(2):383-99. doi:10.1007/s00018014-1679-z.

24. Ren C, Xu K, Liu Z, Shen J, Han F, Chen Z, et al. 2015. Dual-reporter surrogate systems for efficient enrichment of genetically modified cells. No. 72. p 2763-72.

25. Bai Y, He L, Li P, Xu K, Shao S, Ren C, et al. Efficient genome editing in chicken DF-1 cells using the CRISPR/Cas9 system. G3 (Bethesda, Md.). 2016;6(4): 917-23. doi:10.1534/g3.116.027706.

26. Lyu M, Shalitana A, Luo J, He H, Sun S, Wang P. Overexpression of the Tuberous sclerosis complex 2 (TSC2) gene inhibits goat myoblasts proliferation and differentiation in understanding the underlying 
mechanism of muscle development. Gene. 2020;757:144943. doi:10.1016/j.gene.2020.144943.

27. Tian H, Luo J, Zhang Z, Wu J, Zhang T, Busato S, et al. 2018. CRISPR/Cas9-mediated Stearoyl-CoA desaturase 1 (SCD1) deficiency affects fatty acid metabolism in goat mammary epithelial cells. No. 66. p 10041-52.

28. Zhang Z, Li J, Guan D, Liang C, Zhuo Z, Liu J, et al. A newly identified IncRNA MAR1 acts as a miR$487 \mathrm{~b}$ sponge to promote skeletal muscle differentiation and regeneration. Journal of cachexia sarcopenia muscle. 2018;9(3):613-26. doi:10.1002/jcsm.12281.

29. Gao Y, Wu H, Wang Y, Liu X, Chen L, Li Q, et al. Single Cas9 nickase induced generation of NRAMP1 knockin cattle with reduced off-target effects. Genome biology. 2017;18(1):13. doi:10.1186/s13059016-1144-4.

30. Moretti A, Fonteyne L, Giesert F, Hoppmann P, Meier AB, Bozoglu T, et al. Somatic gene editing ameliorates skeletal and cardiac muscle failure in pig and human models of Duchenne muscular dystrophy. Nature medicine. 2020;26(2):207-14. doi:10.1038/s41591-019-0738-2.

31. Yan N, Sun Y, Fang Y, Deng J, Mu L, Xu K, et al. A universal surrogate reporter for efficient enrichment of CRISPR/Cas9-Mediated Homology-Directed repair in mammalian cells. Molecular therapy. Nucleic acids. 2020;19:775-89. doi:10.1016/j.omtn.2019.12.021.

32. Seki A, Rutz S. Optimized RNP transfection for highly efficient CRISPR/Cas9-mediated gene knockout in primary T cells. The Journal of experimental medicine. 2018;215(3):985-97. doi:10.1084/jem.20171626.

33. Liu J, Gaj T, Yang Y, Wang N, Shui S, Kim S, et al. Efficient delivery of nuclease proteins for genome editing in human stem cells and primary cells. Nature protocols. 2015;10(11):1842-59. doi:10.1038/nprot.2015.117.

34. Malumbres M, Barbacid M. Mammalian cyclin-dependent kinases. Trends Biochem Sci. 2005;30(11):630-41.

35. Bertoli C, Skotheim JM, de Bruin RAM. Control of cell cycle transcription during G1 and S phases. Nat Rev Mol Cell Biol. 2013;14(8):518-28. doi:10.1038/nrm3629.

36. Hwang J, Kim AR, Kim KM, II Park J, Oh HT, Moon SA, et al. TAZ couples Hippo/Wnt signalling and insulin sensitivity through Irs1 expression. Nature communications. 2019;10(1):421. doi:10.1038/s41467-019-08287-x.

37. Rodrigues Alves APN, Fernandes JC, Fenerich BA, Coelho-Silva JL, Scheucher PS, Simões BP, et al. 2019. IGF1R/IRS1 targeting has cytotoxic activity and inhibits PI3K/AKT/mTOR and MAPK signaling in acute lymphoblastic leukemia cells. No. 456. p 59-68.

38. Wang G, Pan J, Zhang L, Wei Y, Wang C. Long non-coding RNA CRNDE sponges miR-384 to promote proliferation and metastasis of pancreatic cancer cells through upregulating IRS1. Cell proliferation. 2017;50(6). doi:10.1111/cpr.12389.

39. Motohashi N, Alexander MS, Shimizu-Motohashi Y, Myers JA, Kawahara G, Kunkel LM. Regulation of IRS1/Akt insulin signaling by microRNA-128a during myogenesis. Journal of cell science. 2013;126(Pt 12):2678-91. doi:10.1242/jcs.119966. 


\section{Tables}

Table 1 Primer information for miRNA and mRNA quantitative reverse transcription

\begin{tabular}{|c|c|c|}
\hline Gene & Primer name & Primer sequence $\left(5^{\prime}\right.$ to $\left.3^{\prime}\right)$ \\
\hline \multirow[t]{3}{*}{$\begin{array}{l}\operatorname{miR}-487 b- \\
3 p\end{array}$} & Stem-loop & $\begin{array}{l}\text { GTCGTATCCAGTGCAGGGTCCGAGGTATTCG } \\
\text { CACTGGATACGACAAGTGG }\end{array}$ \\
\hline & miR-487b-3p-F & CGGGCAATCGTACAGGGT \\
\hline & miR-487b-3p-R & CAGTGCAGGGTCCGAGGTAT \\
\hline \multirow[t]{2}{*}{ 18S-rRNA } & $\begin{array}{l}\text { 18S-rRNA- } \\
\text { qPCR-F }\end{array}$ & GTGGTGTTGAGGAAAGCAGACA \\
\hline & $\begin{array}{l}\text { 18S-rRNA- } \\
\text { qPCR-R }\end{array}$ & TGATCACACGTTCCACCTCATC \\
\hline \multirow[t]{2}{*}{ IRS1 } & IRS1-F & GTAGTGGCAAACTCCTGTCTTGT \\
\hline & IRS1-R & GAGTAGTAGGAGAGGACGGGCT \\
\hline \multirow[t]{2}{*}{ PCNA } & PCNA-F & CGCTTAAGGATCTCATCAATGAG \\
\hline & PCNA-R & GTTACGGTCGCAGCGGTAAG \\
\hline \multirow[t]{2}{*}{ Cyclin E } & Cyclin E-F & СТСССТGАТTСССАСАССТG \\
\hline & Cyclin E-R & CATAAGATGCTTGTCCCTCA \\
\hline \multirow[t]{2}{*}{ CDK2 } & CDK2-F & TCATGGATGCCTCTGCACTC \\
\hline & CDK2-R & CTCTGGCTAGTCCGAAGTCTG \\
\hline \multirow[t]{2}{*}{ p27 } & $\mathrm{p} 27-\mathrm{F}$ & CGGCGGTGCCTTTACTT \\
\hline & $\mathrm{p} 27-\mathrm{R}$ & GCAGGTCGCTTCCTTATCC \\
\hline \multirow[t]{2}{*}{ GAPDH } & GAPDH-F & CCACGCCATCACTGCCACCC \\
\hline & GAPDH-R & CAGCCTTGGCAGCGCCAGTA \\
\hline
\end{tabular}

Table 2 The primers used to construct the plasmid 


\begin{tabular}{|c|c|c|}
\hline Name & Primer name & Primer sequence $\left(5^{\prime}\right.$ to $\left.3^{\prime}\right)$ \\
\hline \multirow[t]{4}{*}{ sgRNA1 } & $\operatorname{sg} 1-\mathrm{F}$ & сасcCGCTCTTGATACTGAAAAAG \\
\hline & sg1-R & aаacCTTTTTCAGTATCAAGAGCG \\
\hline & sg1-SR-F & gatcCGCTCTTGATACTGAAAAAGTGG \\
\hline & sg1-SR-R & ggccCCACTTTTTCAGTATCAAGAGCG \\
\hline \multirow[t]{4}{*}{ sgRNA2 } & sg2-F & сасCTACTCATGTCGAATCGTACA \\
\hline & sg2-R & aaacTGTACGATTCGACATGAGTA \\
\hline & sg2-SR-F & gatcTACTCATGTCGAATCGTACAGGG \\
\hline & sg2-SR-R & ggccCCCTGTACGATTCGACATGAGTA \\
\hline \multirow[t]{4}{*}{ sgRNA3 } & sg3-F & сасcCGCTCTTGATACTGAAAAAG \\
\hline & sg3-R & aaacCTTTTTCAGTATCAAGAGCG \\
\hline & sg3-SR-F & gatcCGCTCTTGATACTGAAAAAGTGG \\
\hline & sg3-SR-R & ggccCCACTTTTTCAGTATCAAGAGCG \\
\hline \multirow[t]{2}{*}{ Wild-IRS1 } & Wild-IRS1-F & cggctcgagCAGCAAATСCTССТTTAАСТC \\
\hline & Wild-IRS1-R & aatgcggccgcGCACGATATACAACGTGCAG \\
\hline \multirow[t]{2}{*}{ Mutant-IRS1 } & Mutant-IRS1-F & CTCAGTAGATGGGCTAATGCACCC \\
\hline & Mutant-IRS1-R & GAAATGGGTGCCGATTACCATCTAC \\
\hline
\end{tabular}

\section{Figures}
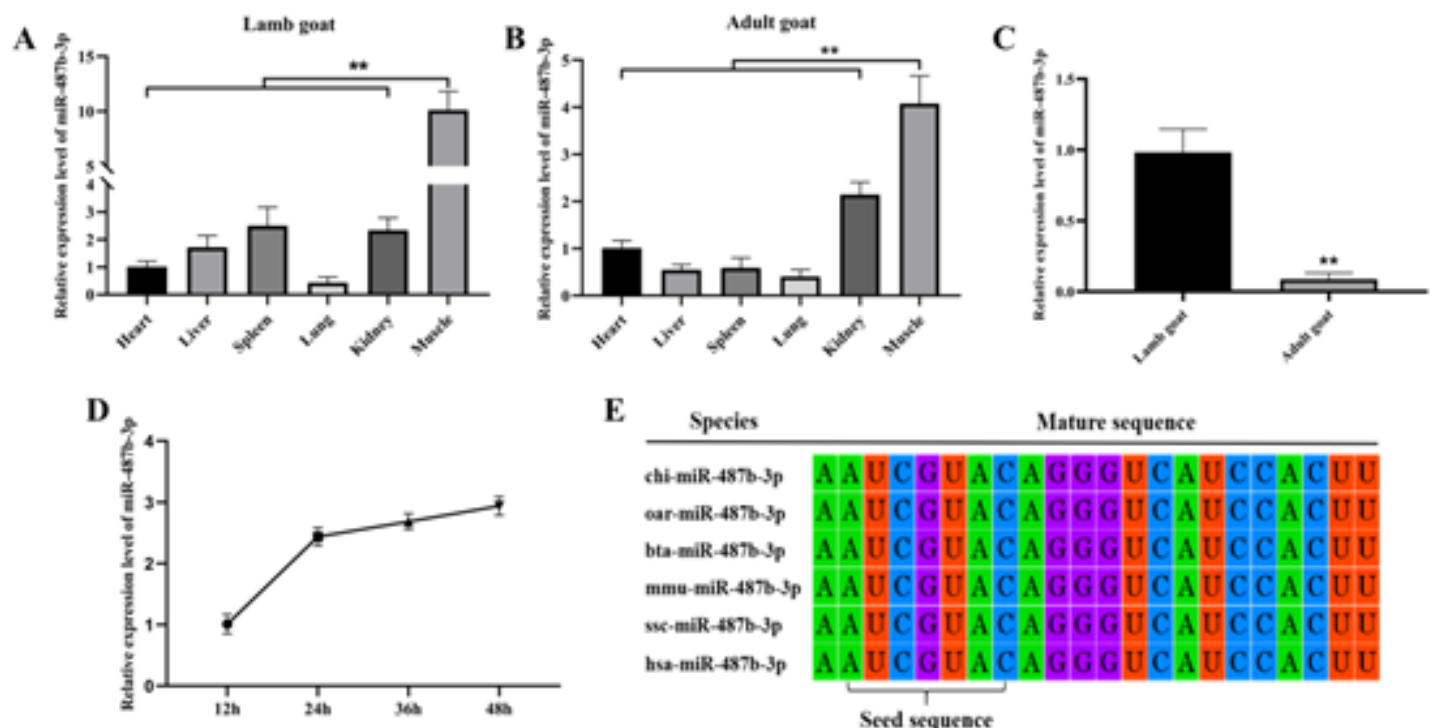

E

Mature sequence

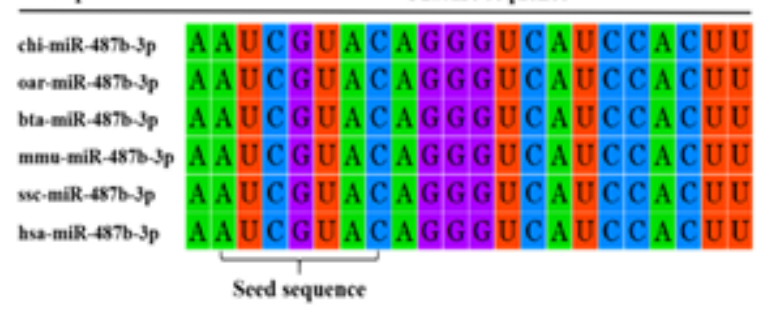

Figure 1 
miR-487b-3p is a candidate regulator in goat myogenesis. a The expression level of miR-487b-3p in different tissues of lamb goat. b The expression level of miR-487b-3p in different tissues of adult goat. c Differential expression of miR-487b-3p between lamb and adult goat muscle tissue. $d$ Up-regulation of miR-487b-3p during goat myoblast proliferation. e Comparation of miR-487b-3p seed sequences from Ovis aries, Bos taurus, Mus musculus, Sus scrofa and Homo sapiens.

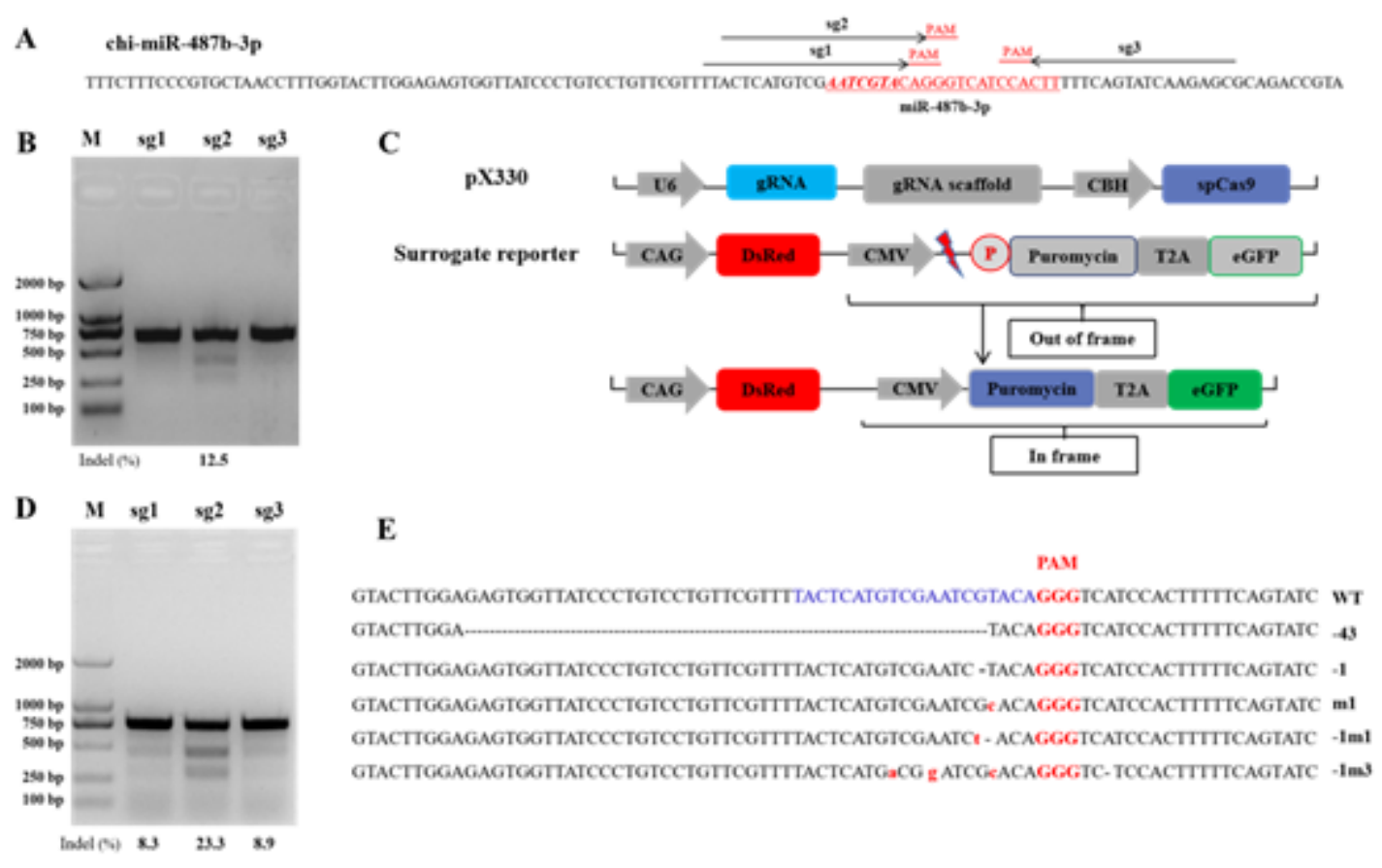

Figure 2

sgRNA target sites for miR-487b-3p knockout and RPG-based enrichment of genetically targeted goat myoblast cells. a miR-487b-3p genome sequence and sgRNA design. The three sgRNAs (sg1, sg2, sg3) are indicated by arrows with the PAM motifs by red line. The miR-487b-3p mature sequence is underlined and highlighted in red font with the seed sequence italic. $b$ The frequencies of indels induced by the three sgRNAs assessed by T7E1-cleavage assay with the bands densitometry measured by Image J. c Schematic diagram of the RPG surrogate reporter system, including a sgRNA/Cas9 expression vector and a DsRed-Puror-eGFP (RPG) surrogate reporter. $d$ The frequencies of indels induced by the three sgRNAs after the RPG surrogate reporter-based screening. e Representative random indels adjacent to PAM sequence from the sg2 experiment group. The miR-487b-3p mature sequence is indicated in blue font. The PAM motif of sg2 is highlighted in red font. Mutated bases are indicated in red and lowercase font. wild type (WT), deletion (-) and mutation (m) are shown to the right of each sequence. 
PAM

A GTACTTGGAGAGTGGTTATCCCTGTCCTGTTCGTTTTACTCATGTCGAATCGTACAGGGTCATCCACTTTTTCAGTATC WT GTACTTGGAGAGTGGTATCCCTGTCCTGTTCGTTT TACTCATGTCG AATCGT _............... CATCCACTTTTTCAGTATC -7
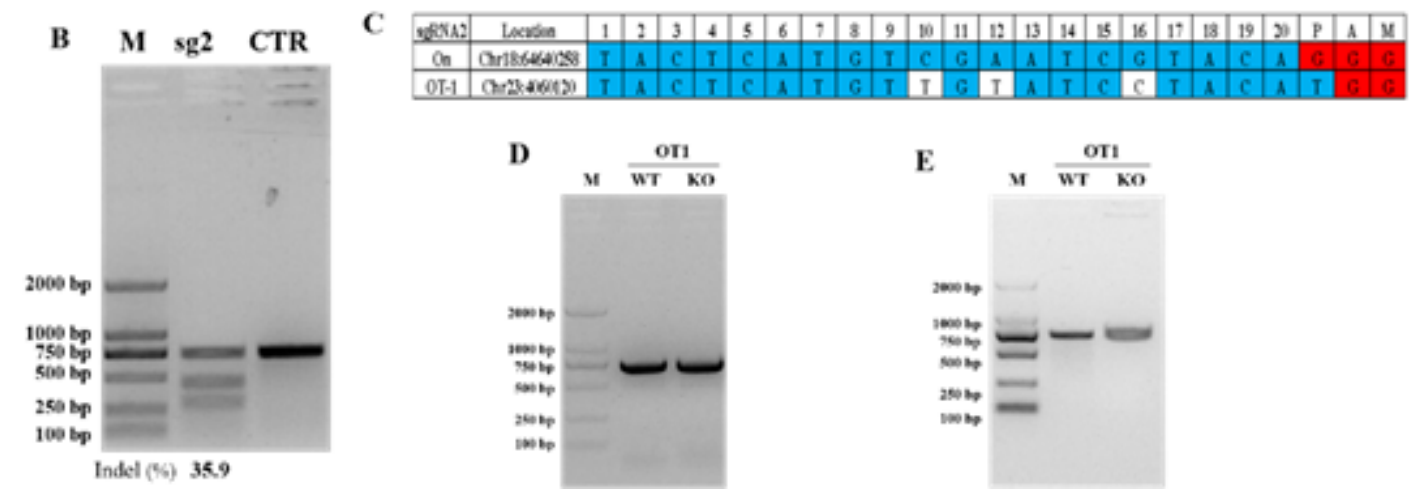

\section{Figure 3}

The miR-487b-3p KO cell clone and off-target effect analyses. a Sequencing result of the miR-487b-3p KO goat myoblast cell clone with the deletion of 7 nucleotides. b T7E1 assay result for expanded miR-487b$3 p$ KO cell clone. $c$ The potential off-target site for sg2 predicted. $d$ Detection of the off-target site genomic sequence by PCR. e T7E1 assay result showed no difference between the WT and miR-487b-3p $\mathrm{KO}$ cells at the potential off-target site. OT, off-target; WT, wild type of each off-target site.
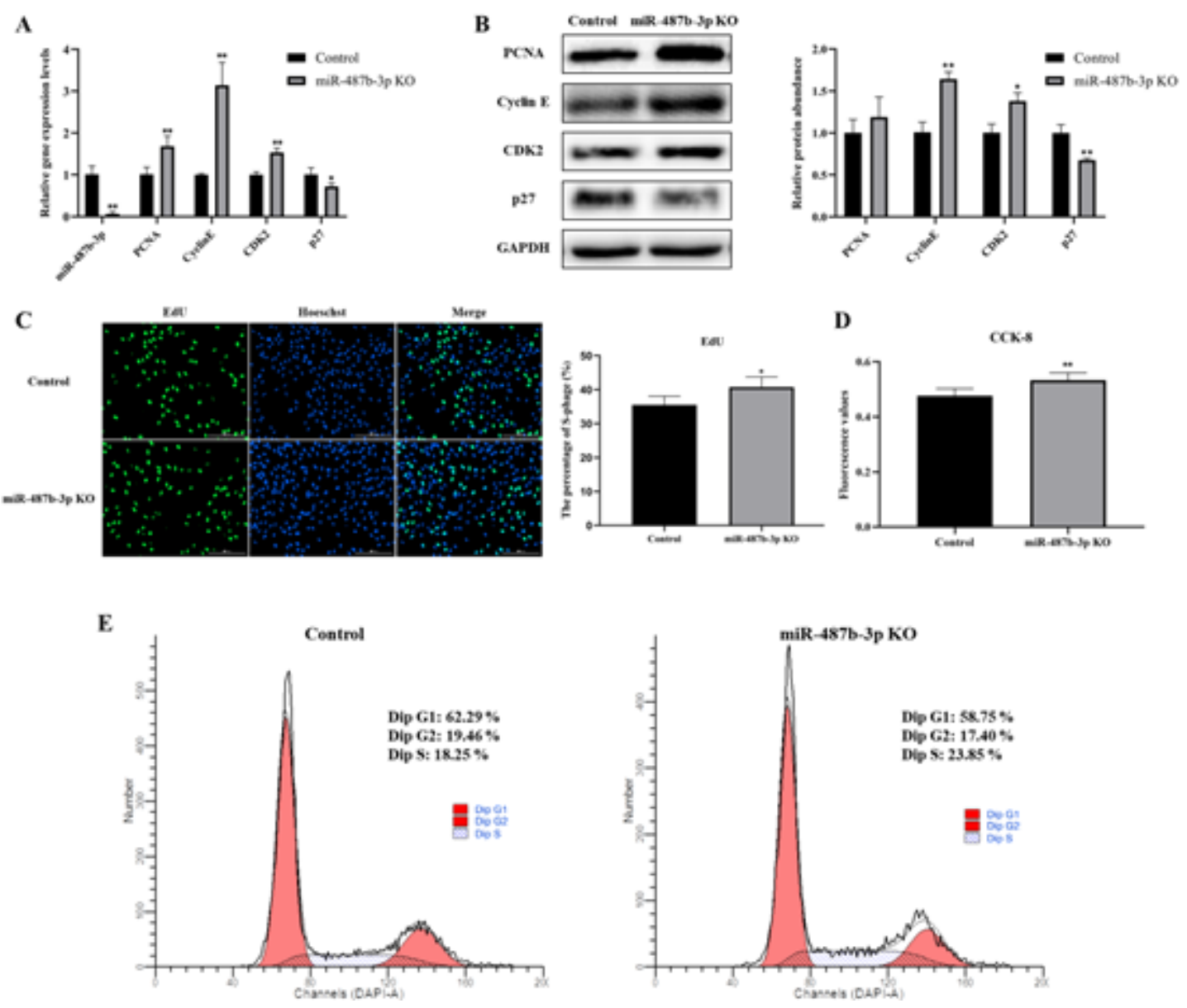

Figure 4 
Knockout of miR-487b-3p promotes goat myoblast cell proliferation. a RT-qPCR analysis result of the expression levels of miR-487b-3p and cell cycle-related genes. b Western blot analysis result of PCNA, Cyclin E, CDK2 and p27 in the wild type control and miR-487b-3p KO cells. c EdU assay result for comparing the wild type control and miR-487b-3p KO cells. Cells during DNA replication were stained by EdU (green) and cell nuclei were stained with Hoechst (blue). The percentage of EdU positive cells/Hoechst positive cells (total cells) was quantified. $d$ Cell viability was detected by cell count kit 8 (CCK-8) for comparing the wild type control and miR-487b-3p KO cells. The result was represented by the absorbance value at $450 \mathrm{~nm}$ after incubation with 10\% CCK-8 solution for $4 \mathrm{~h}$. e Cell cycle analysis for comparing the wild type control and miR-487b-3p KO cells. Data was shown by mean \pm SD of three independent experiments. ${ }^{*}, \mathrm{P}<0.05 ; * *, \mathrm{P}<0.01$

A psi-CHENKTM-2

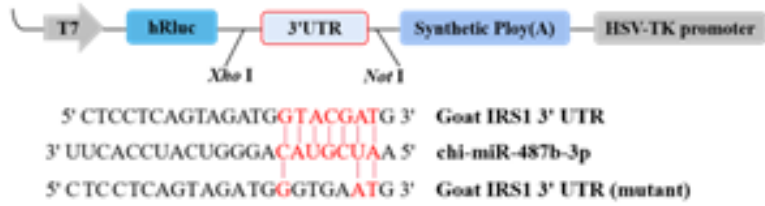

B

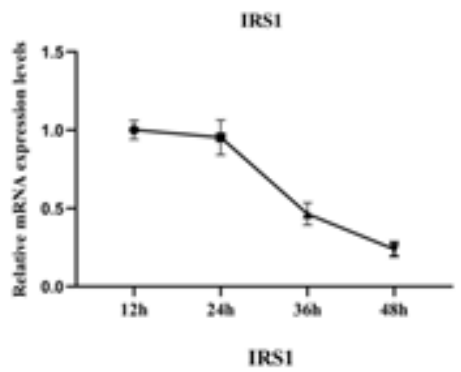

C

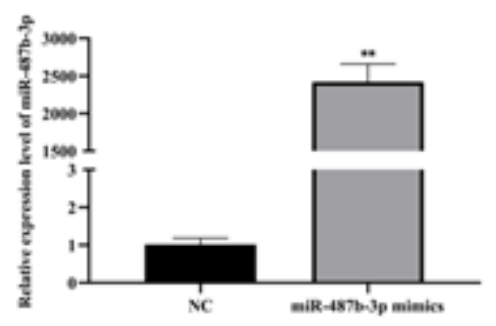

D

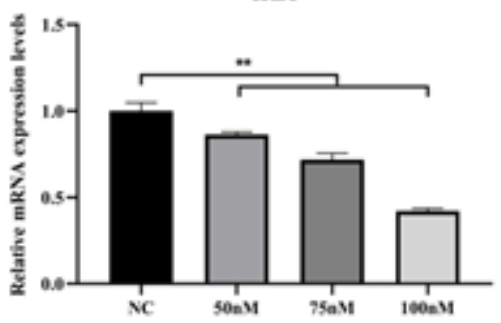

E

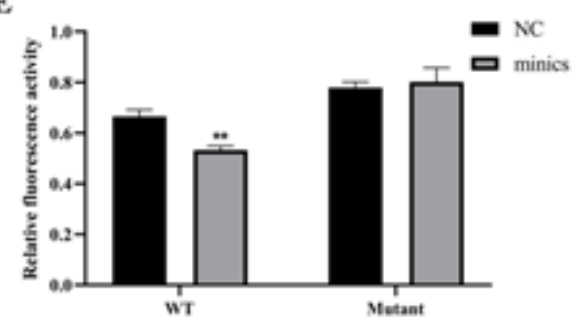

$\mathbf{F}$

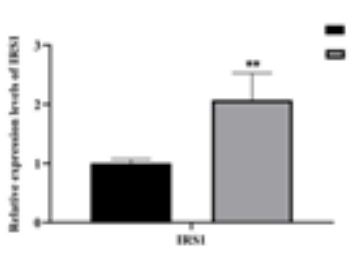

- Comed

G

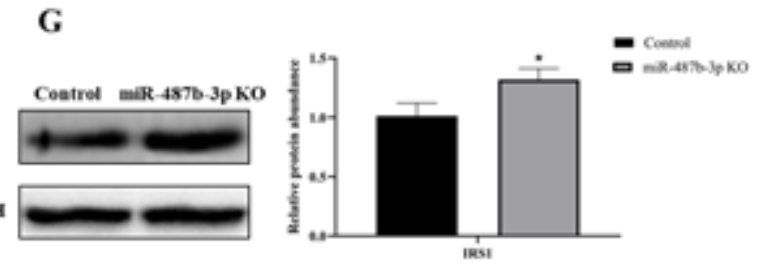

Figure 5

miR-487b-3p directly target IRS1 3'UTR. a The construct of the dual-luciferase reporter with goat IRS1 $3^{\prime} U T R$ and the predicted wildtype or mutant miR-487b-3p binding sites. $b$ The expression profile of IRS1 mRNA during goat myoblast proliferation. c IRS1 mRNA expression inhibited as responded to different concentration of miR-487b-3p mimics. NC, negative control miRNA mimics. $d$ The quantification of miR$487 b-3 p$ in goat myoblast cells after transfected with the mimics. e The results of the dual-luciferase reporter assay. Cells were co-transfected with the dual-luciferase reporter containing the wildtype (WT) or mutant IRS1 3'UTR, either with the negative control or miR-487b-3p mimics. The activity of renilla luciferase was normalized to firefly luciferase. $f$ IRS1 mRNA expression detected by RT-qPCR in the 
control wildtype and miR-487b-3p KO cells. g IRS1 protein expression detected by western blot in the control wildtype and miR-487b-3p KO cells. Each treatment was carried out in triplicate and repeated 3 times. Data was shown by mean \pm SD of three independent experiments. ${ }^{*}, \mathrm{P}<0.05 ; * *, \mathrm{P}<0.01$

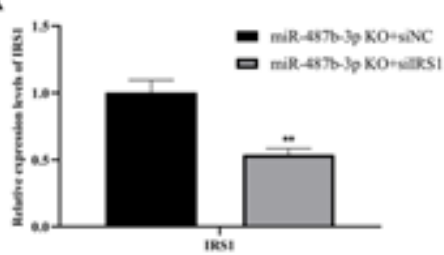

c

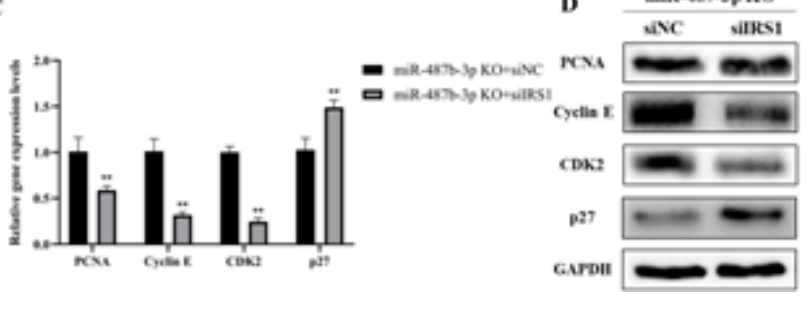

B
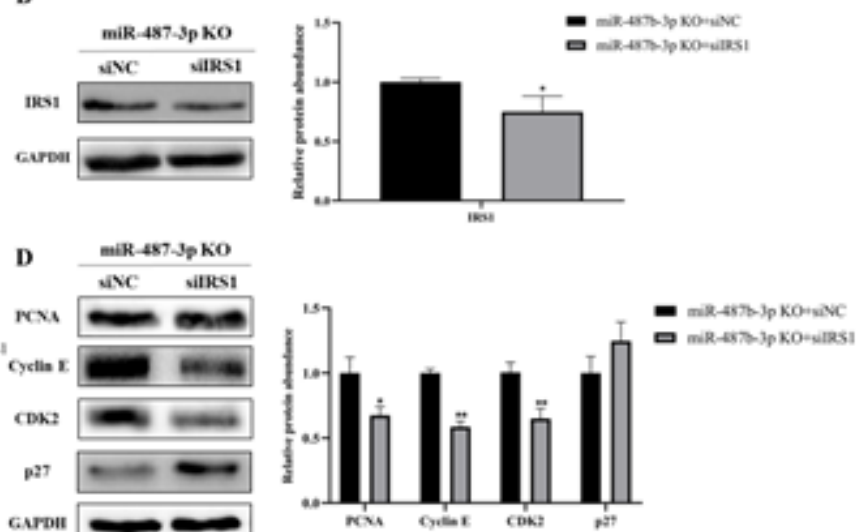

Figure 6

miR-487b-3p KO affects myoblast cells proliferation by targeting IRS1. a IRS1 mRNA expression in miR487b-3p KO cells transfected with siNC or silRS1. b IRS1 protein expression in miR-487b-3p KO cells transfected with siNC or silRS1. c The mRNA expression of cell cycle related-genes in miR-487b-3p KO cells transfected with siNC or silRS1. $d$ The protein expression of cell cycle related-genes in miR-487b-3p $\mathrm{KO}$ cells transfected with the siNC or silRS1. Each treatment was carried out in triplicate and repeated 3 times. Data was shown by mean $\pm \mathrm{SD}$ of three independent experiments. ${ }^{*}, \mathrm{P}<0.05 ; * \star, P<0.01$

\section{Supplementary Files}

This is a list of supplementary files associated with this preprint. Click to download.

- SupplementaryMaterial.docx 